\title{
RF SYSTEM DESIGN FOR THE EMMA FFAG
}

\author{
C.D.Beard ${ }^{\#}$, N.Bliss, S.A.Griffiths, C.Hill, P.A.McIntosh, A.Moss, A.Wheelhouse, C. White, STFC \\ Daresbury Laboratory, Warrington, WA4 4AD, UK \\ D.Teytelman, DimTel, San Jose, California, USA
}

\section{Abstract}

In this report the RF system design for EMMA (Electron Machine with Many Applications) is described. The power source options, power supplies, waveguide distribution scheme and control system is discussed. The architecture necessary to meet the operation specifications requires a large degree of adjustment and flexibility to accommodate machine commissioning requirements. To simplify commissioning and enhance the versatility of the machine, a complex RF system is desired. This report details the RF "knobs" included to meet this requirement.

\section{INTRODUCTION}

EMMA[1] is a prototype non-scaling FFAG (Fixed Field Alternating Gradient) facility currently under construction at Daresbury Laboratories. The accelerator will utilise ALiCE (Accelerators and Lasers in Combined Experiments) [2] (formerly known as the Energy Recovery Linac Prototype, ERLP) as its injector, and for ease of synchronising and minimising the size of the RF cavities, $1.3 \mathrm{GHz}$ has been chosen as the operating frequency for EMMA. The injected beam will be $10 \mathrm{MeV}$ and will be extracted at $20 \mathrm{MeV}$. A layout of the accelerator complex is shown in Figure 1 below.

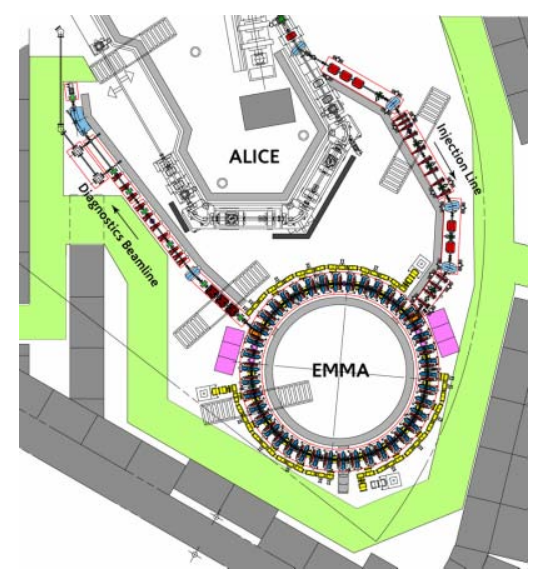

Figure 1: Accelerator Layout

For this 42-cell machine and due to the fast acceleration required for this accelerator, 19 cavities are located in alternate cells, by omitting 2 cavities, leaving space for beam injection and extraction. Since this is a demonstrator machine, the RF system has to be robust in order to test the whole parameter space, which includes varying the RF frequency and the rate of acceleration. Initially an acceleration of $2.3 \mathrm{MV}$ per turn with the potential to upgrade to a maximum of $3.4 \mathrm{MV}$ is also desirable. The frequency range for operation is $5.5 \mathrm{MHz}$, and stable RF phase and amplitude control is required to ensure stable operation.

The full RF parameters are listed in Table 1 below

Table 1: RF design parameters for EMMA.

\begin{tabular}{|l|c|}
\hline Machine Parameters & Value \\
\hline Frequency (GHz) & 1.3 \\
\hline Number of Straights & 21 \\
\hline Number of Cavities & 19 \\
\hline Total Acc per turn & $2.3 \mathrm{MV}$ \\
\hline Upgrade Acc per turn & $3.4 \mathrm{MV}$ \\
\hline Beam Aperture & $40 \mathrm{~mm}$ \\
\hline RF Pulse Length & $1.6 \mathrm{~ms}$ \\
\hline RF Repetition rate & $1 \mathrm{to} 20 \mathrm{~Hz}$ \\
\hline
\end{tabular}

A tender exercise is currently underway to procure a system to that meets the RF design parameters in table 1 . The final system may vary considerably, once this exercise has been completed.

\section{RF CAVITY}

In order to provide acceleration from $10 \mathrm{MeV}$ to 20 $\mathrm{MeV}$ an RF cavity is required that is capable of producing up to $180 \mathrm{kV}$ accelerating voltage. For the cavity design optimisation, there were some geometrical restraints which reduced the potential performance of the cavity. EMMA is very compact by nature and therefore the total longitudinal length allowed was only $110 \mathrm{~mm}$ flange to flange, whilst the beam aperture had to be greater than 40 $\mathrm{mm}$ diameter. This reduced the possible efficiency for a normal conducting cavity, and so a design with the highest possible $\mathrm{Q}$ was sought.

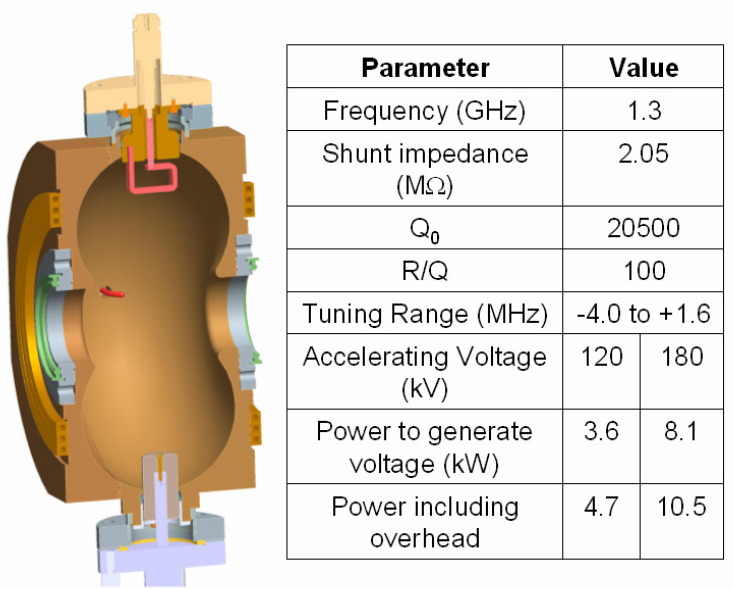

Figure 2: Final Cavity Design and Parameters 
The final cavity design is shown in Figure 2 alongside the cavity operating parameters. Niowave Inc, has recently been contracted to supply all 19 (+ 1 spare) cavities for the EMMA accelerator and further details of the design, manufacturing and test results can be found in a separate paper at this conference [3].

\section{RF DISTRIBUTION}

In order to distribute equal amounts of RF power to each of the cavities, allowing control of both phase and amplitude, a robust RF distribution system has been proposed. Since there are constraints due to size and the need to have crane access to the machine, numerous schemes have been considered. A cascaded system has been found to be the most suitable, whereby sufficient RF power is tapped off the distribution network around the circumference of the ring, requiring increasing levels of coupling towards the end of the waveguide or coaxial lines. Since we have not yet decided upon the final choice for the RF power source, we can either have a single device or a number of devices situated around the ring. The level of coupling required along the distribution is displayed in Table 2, which shows how the coupling changes along the section and the desired split ratio.

\section{Table 2: RF Distribution ratio}

\begin{tabular}{|l|l|l|l|l|l|}
\hline 2 & 3 & 4 & 5 & 6 & 7 \\
\hline $50 \%$ & $33 \%$ & $25 \%$ & $20 \%$ & $16.6 \%$ & $14.3 \%$ \\
\hline $3 \mathrm{~dB}$ & $4.8 \mathrm{~dB}$ & $6 \mathrm{~dB}$ & $7 \mathrm{~dB}$ & $7.8 \mathrm{~dB}$ & $8.4 \mathrm{~dB}$ \\
\hline
\end{tabular}

The benefit of using the cascaded approach is that depending on the number of power sources, it would be easy to reconfigure for any number of configurations, dependant on the power capabilities of the source.

An example of this Figure 3 demonstrates one possible solution with an IOT providing RF power to a section of the waveguide structure coupling power to 3 cavities.

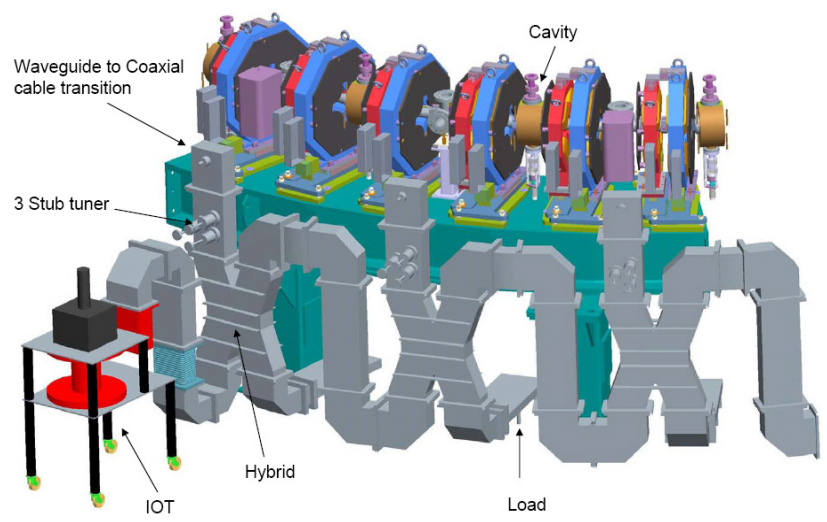

Figure 3: Proposed section of waveguide distribution

The proposed solution adopts hybrids to split a known percentage of RF power to the cavity with the rest of the power feeding the remaining cavity string. This allows for removal of any reflected RF power direct to a suitable load, with motorised 3-stub tuners proposed to allow remote tuning of the RF phase to the time of flight of the particles as the cavity frequency is varied. Other components required per cavity include directional couplers, WR650 to coaxial transitions and a number of $\mathrm{H}$ and $\mathrm{E}$ Bends.

\section{RF POWER SOURCES AND PRE-AMPS}

The total peak power required for the EMMA RF system is $90 \mathrm{~kW}$ minimum up to a desirable level of 200 $\mathrm{kW}$. These figures take into account the potential losses in the waveguide distribution and also the overhead required for the low level RF system to be able to control for stable operation. The duty factor for this machine is a maximum of $3.2 \%(1.6 \mathrm{~ms}, 20 \mathrm{~Hz})$, therefore the average power required is a maximum of $6.4 \mathrm{~kW}$.

To deliver this amount of power, a number of options are available at $1.3 \mathrm{GHz}$.. These are;

1. A Single high power device $>120 \mathrm{~kW}$ (e.g. Klystron, IOT or TWT)

2. A number of Lower Power devices $>20 \mathrm{~kW}$ (IOT or TWT)

3. $194 \mathrm{~kW}$ Sources (Solid State Amplifiers, TWT)

Travelling wave tubes (TWT) offer a much larger bandwidth of up to $1 \mathrm{GHz}$, whereas the klystron and IOT would have difficulty meeting the $5.5 \mathrm{MHz}$ bandwidth desired, without the facility of retuning the output circuit of the tube.

For each system there is an associated risk. Using a single device has the inherent risk that should a device fail during operation the time for replacement could be over 1 year.

An option to reduce this risk would be to have a number of smaller power IOTs or TWTs. This would reduce the risk for down time, since these devices are in regular production.

If affordable, the preferred solution would be 19 individual RF amplifiers. This option would provide the optimum for individual phase and amplitude control, and would alleviate the need for the large waveguide distribution.

The cost details for each of these types of system along with the technical ability to meet the specified requirement needs to be fully understood before the system can be procured.

\section{HIGH VOLTAGE POWER SUPPLY (HVPS)}

Until the final RF source has been identified, the final requirement of the HVPS remains unknown. In the instance that the final choice is a single RF source, then it is quite possible that we could use the existing $50 \mathrm{kV} / 16 \mathrm{~A}$ Thales HVPS from the Synchrotron Radiation Source, since this is due to close by September 2008, before EMMA is operational.

In the event that a number of smaller RF sources are implemented, a number a smaller power supplies will be required since the Thales unit is set up for a single device. 
For this reason a modular power supply has been developed in $40 \mathrm{~kW}$ modules that can be used individually or combined in order to power the higher power tubes. A prototype module has been built and tested in order to prove the technique and to measure the operational stability of the device. This modular system has been identified as a much cheaper option than conventional techniques that adopt a thyratron, and therefore validation of the design is critical.

\section{LOW LEVEL RF AND SYNCHRONISATION}

Beam storage time in EMMA will range from 10 to 1000 turns or, at $12.3 \mathrm{~ns}$ per revolution from 0.12 to 12.3 $\mu \mathrm{s}$. Providing feedback-based beam loading compensation with such a short storage time demands unrealistically wide closed loop bandwidth. The studies carried out so far only consider stabilization of cavity voltages to reject HVPS disturbances, cavity tuning errors, and cavity voltage measurement noise. The beam will sample the residual errors in cavity voltage stabilization on the pulseby-pulse basis.

The main challenges in implementing Low Level RF (LLRF) control in EMMA are:

1. Frequency tuning,

2. Injection phase control,

3. Rejection of HVPS ripple,

4. Cavity voltage stabilization in presence of cavity tuning errors,

5. Sensitivity to vector sum gain, phase, and delay errors,

6. Cavity vector sum calibration and tracking.

In order to assess these, a seven-cavity RF station has been modelled. Using non-IQ LLRF processing demonstrates excellent reference tracking, loop stability and robustness under cavity detuning. These results strongly exceed the practically required range.

A Simulink model was assessed using the proposed LLRF system design detailed in Figure 4 .

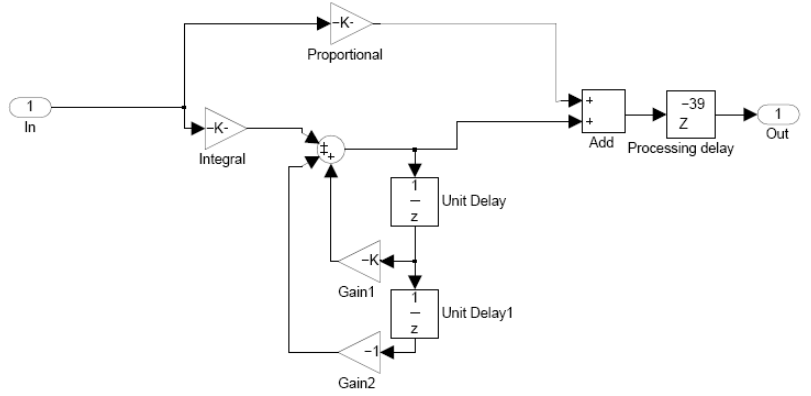

Figure 4: Simulink model of the feedback controller I

With integrated self-calibration, RF reference tracking, and cavity frequency measurement, the LLRF processing is expected to reliably meet a field stability of $1 \%$ amplitude and 1 degree phase levels for the worst case scenario during large frequency shifts from the desired frequency of $1.3 \mathrm{GHz}$.
Figure 5 shows the Simulink modelling results for the 7 cavity system for the worst case scenario for both amplitude and phase errors.
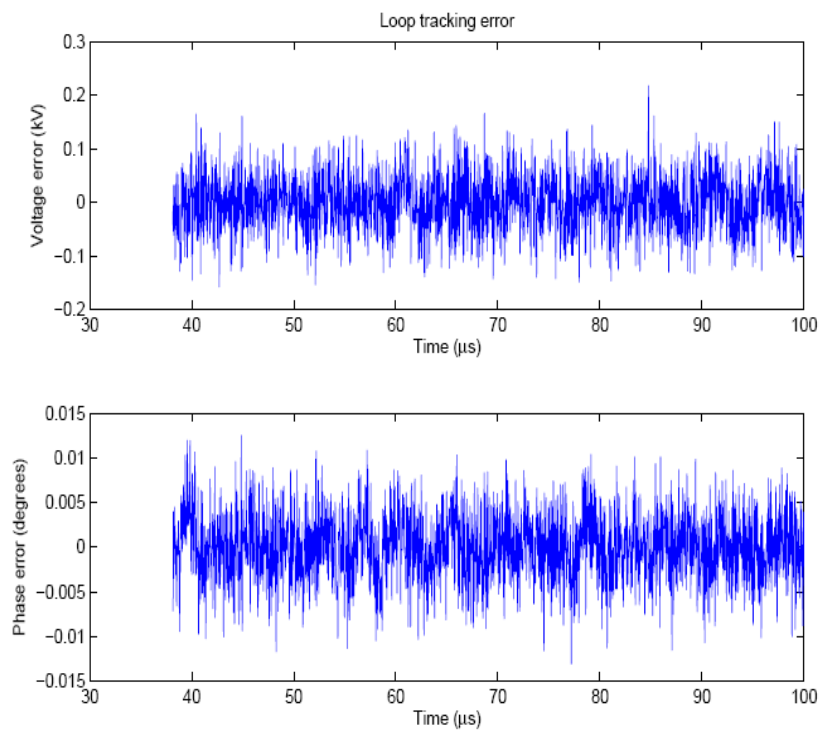

Figure 5: Simulation results showing phase and amplitude errors

\section{CONCLUDING REMARKS}

Evaluation of the RF system has now been completed, demonstrating possible solutions to all parts of the system from power supply to stable RF power delivered to each cavity. The final system is dependant upon the outcome of the tender exercise and an affordable solution determined. The tender exercise for the EMMA RF system has finished an evaluation of the performance and costs of the proposed solutions are underway. Once this has been resolved the procurement of the RF system is proposed to continue for the next 6 to 9 months.

\section{REFERENCES}

[1] T.R.Edgcock et al, "EMMA: The worlds first nonscaling FFAG" THPP004, EPAC 2008, Geneva.

[2] S.L.Smith et al, "The Status of the Daresbury Energy Recovery Linac Prototype", TUAM02, EPAC 2008, Geneva.

[3] C.D.Beard et al, "EMMA RF Cavity Design and Prototype Testing at Daresbury" THPP003, EPAC 2008, Geneva. 